\title{
Telemedicine: a new frontier for effective healthcare services
}

\author{
S. Scalvini1, M.Vitacca², L. Paletta1', A. Giordano1, B. Balbi2
}

ABSTRACT: Telemedicine: a new frontier for effective healthcare services. S. Scalvini, M.Vitacca, L. Paletta, A. Giordano, B. Balbi.

Telemedicine can be defined as the delivery of healthcare services, where distance is a critical factor, by all healthcare professionals using information and communication technologies for the exchange of valid information for diagnosis, treatment and prevention of disease and injuries, research and evaluation, and for the continuing education of healthcare providers, all in the interest of advancing the health of individuals and their communities. Such a wide definition in-

\begin{abstract}
cludes many health care activities and a large number of applications have been tried, with variable degrees of interaction between all the players in the health care system. This review, starting from the need and opportunity that we are now facing to capitalize the great technological improvements in the field of information and communication technologies to improve also our health services, will illustrate the history, classification and main field of application of Telemedicine. Lastly, the available data on the application of Telemedicine for patients with respiratory diseases will be reviewed. Monaldi Arch Chest Dis 2004; 61: 4, 226-233.
\end{abstract}

Keywords: Telemedicine, tele-health, General Practitioner, COPD, asthma.

1 Co.Me.Te (Consorzio per la Ricerca, Sviluppo e Sperimentazione di Sistemi di Telemedicina), Divisione di Cardiologia e Servizio di Telemedicina, Fondazione S. Maugeri, IRCCS, Gussago (Bs).

2 Divisione di Pneumologia, Fondazione S. Maugeri, IRCCS, Gussago (Bs), Italy.

Correspondence: Simonetta Scalvini, M.D., Servizio di Telecardiologia, Fondazione Salvatore Maugeri, Via Pinidolo 23, 25064 Gussago (Bs), Italy; e-mail: sscalvini@fsm.it

\section{Introduction}

Health care is one of the most important components of any social welfare system. Telemedicine is a very useful and critical application of Information and Communication Technologies (ICTs), and may deliver high-quality health care services regionally, nationally, or globally. Telemedicine enables remote, isolated and rural areas to have clinical support from those hospitals and medical systems with a higher level of medical expertise. It partially relieves the pressure on the medical experts due to their limited number and extends their expertise to needy people in isolated remote locations. With advancements in electronics, communications, software and hardware, it has become possible to transmit many vital parameters to high tech health care centres [1].

Telemedicine is a combination of medical expertise, medical equipment, computer hardware and software and communication technology, by which a patient can be examined, investigated, monitored and treated by a medical expert in distant place. The word "Tele" is a Greek word meaning "distance" and the word "Medicine" derives from the verb "Mederi", a Latin word meaning "to heal". Time Magazine described Telemedicine as "healing by wire" [2]. Hence, telemedicine is "Medicine practiced at a distance" [3]. It was defined in 1998 by WHO (World Health Organization) as "the delivery of healthcare services, where distance is a critical factor, by all healthcare pro- fessionals using information and communication technologies for the exchange of valid information for diagnosis, treatment and prevention of disease and injuries, research and evaluation, and for the continuing education of healthcare providers, all in the interest of advancing the health of individuals and their communities". It can also be defined as "organisation and integration of information technology in such a way that resources outside the local organisation can be used systematically in the activities of health services" [4].

This review, starting from the need and opportunity that we are now facing to capitalise the great technological improvements in the field of ICT to improve our health services, will illustrate the history, classification and main field of application of Telemedicine. Lastly, the available data on the application of Telemedicine for patients with respiratory diseases will be reviewed.

\section{History}

Telemedicine began when long-distance health care services became available and related to telecommunication. The first telephone technology was invented in 1876 by Alexander Graham Bell. After the 1880s, some physicians experimented with telecommunication technologies (such as the telephone, telegraph and wireless) in managing medical practices. Some medical experts used the telephone to accept appointments, or make an online diagnosis in advance, to prevent 
long-term waiting and unnecessary travel. Yet telephone technology could not be developed until World War I, to enhance traditional health care and bring care into the new age. The possibility of long-distance transmission of medical information was established in the 1900s. Willem Einthoven developed the long-distance broadcast of graphic data in 1905 and later widely utilized it in transmitting electrocardiographs in the Presbyterian Hospital of New York in 1910. Moreover, longdistance diagnosis had been used in communication between rural physician and urban hospitals after World War I.

A well-known telemedicine project was established in the 1960s when the University of $\mathrm{Ne}$ braska transmitted neurological examinations across its campus for medical purposes. Moreover, the University demonstrated a telemedicine link 112 miles away with the state hospital (Norfolk State Hospital, Nebraska State), which provided speech therapy, neurological examinations, diagnosis for difficult psychiatric cases, case consultations, research seminars and education and training [5].

The National Aeronautics and Space Administration (NASA) pioneered the satellite communication required to demonstrate telemedicine services in the 1960s. NASA tested satellite-based communication to remote areas where it was difficult to receive radio waves, including spacecraft, aircraft, mountains, and valleys. From the early 1970 s to the late 1980 s, digital communication techniques became common and telemedicine improved in quality, accuracy, convenience and cost. During 1995 and 1997, the U.S.A. Department of Defence established telemedicine links via satellite that connected the battlefield with several different medical centres in the European continent, America, and nearby aircraft carriers. The system reduced referral times and saved lives. The capabilities of this project included computerised medical records, full-motion remote video consultation between medical units and tertiary care facilities, forward delivery of laboratory and radiology results and prescriptions, digital diagnostic devices (such as ultrasound and film less teleradiology), and medical command and control technologies.

\section{Classification}

Telemedicine can be classified on the basis of the mode of operation or on the basis of application. According to the mode of operation it can be divided into two groups: real time (interactive) mode and store-and-forward mode. In real-time interactive mode, the patient is present with an attending physician or paramedical personnel, and a specialist is present at a remote medical centre. In the store-and-forward mode, all relevant information (data, graphics, images, etc.) is transmitted

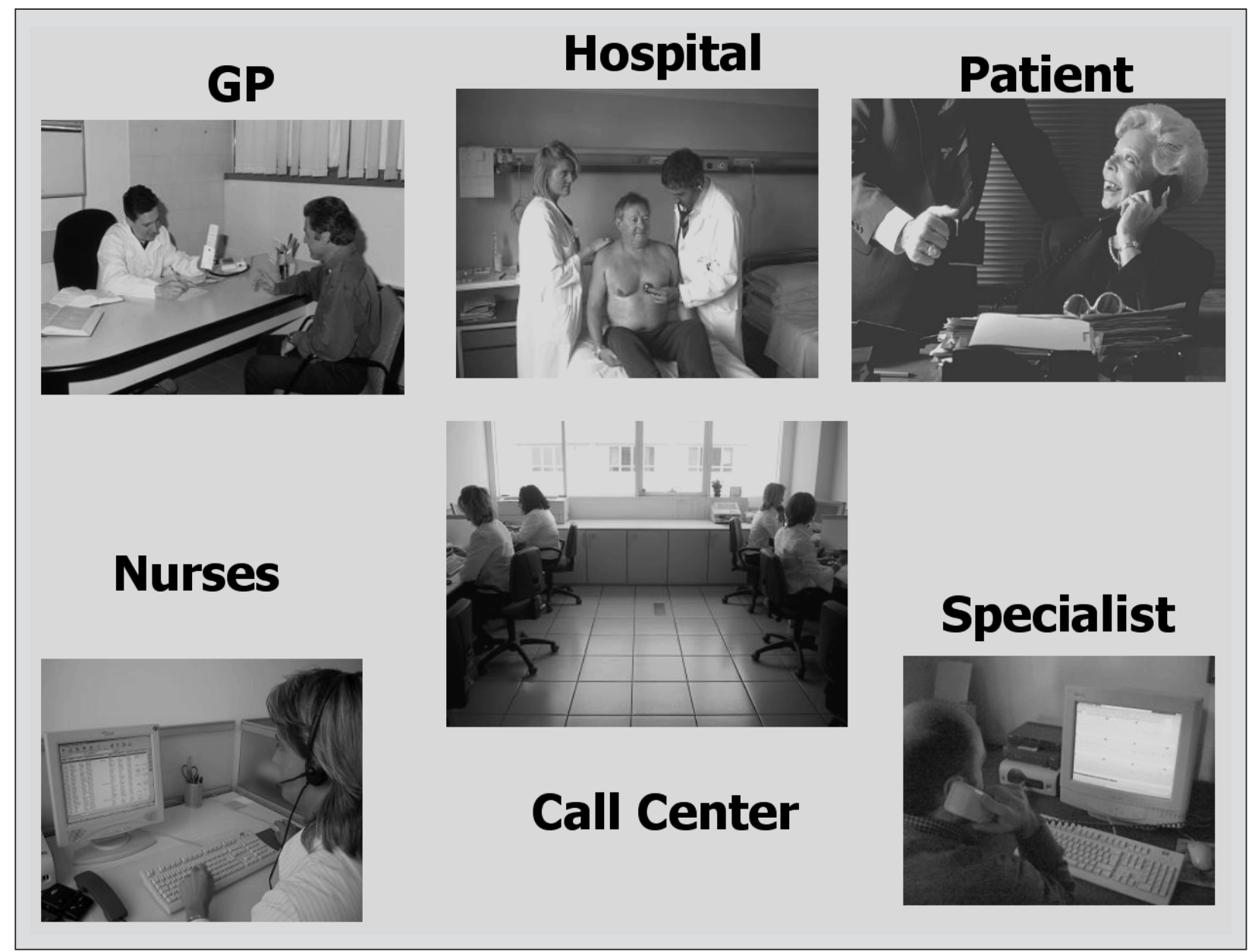

Fig. 1. - Telemedicine: the players. 


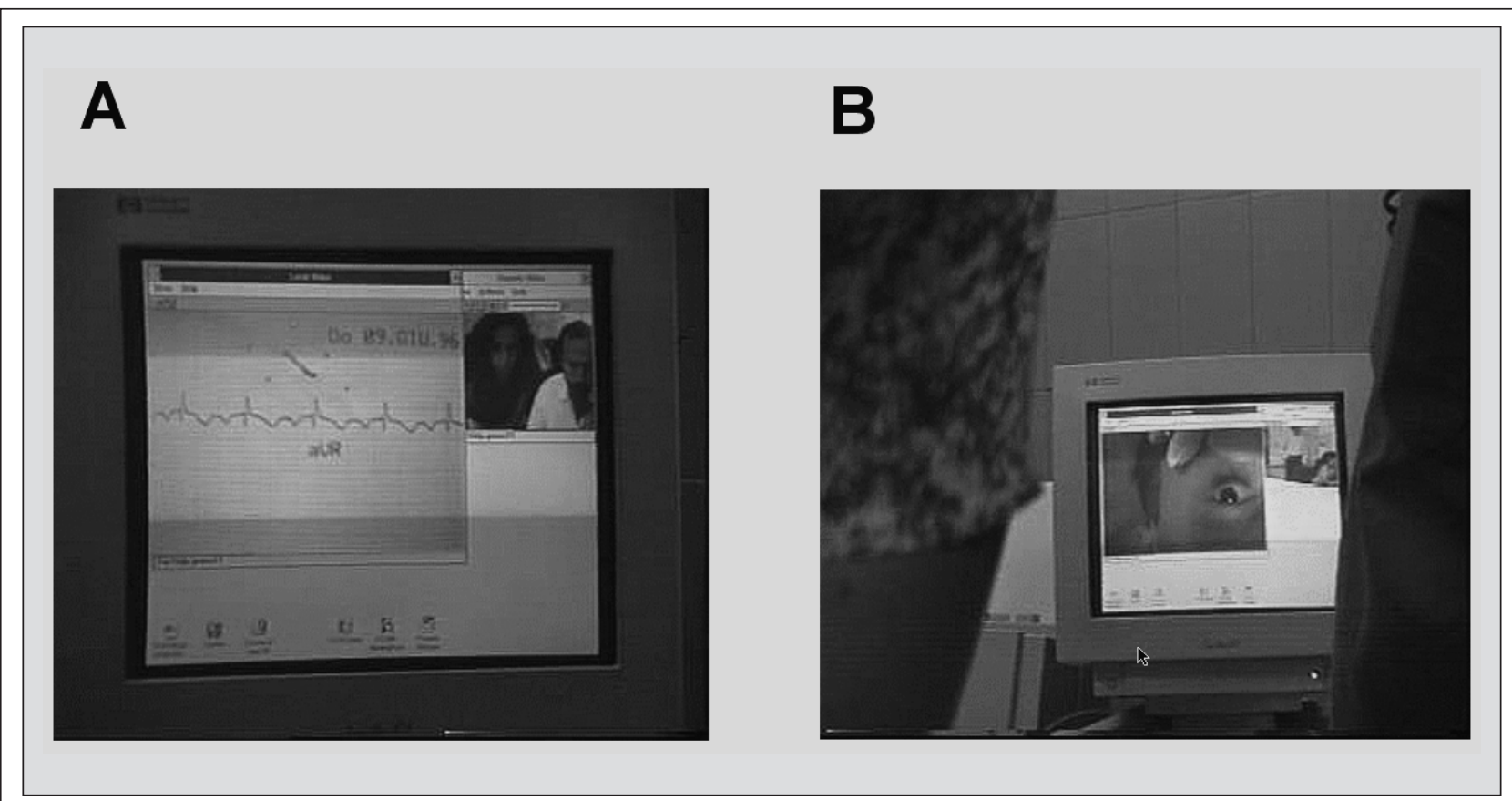

Fig. 2. - Examples of teleconsultations. A: telematic transmission of EKG, B: Toxicologic consultation with the aid of patient's images.

electronically to the specialist. For this mode, the response is not immediate, and in most cases, a few hours or even days elapse before the specialist's report is received. Telemedicine application includes activities such as teleconsultation, telediagnosis applied to different areas of intervention such as telepathology, telepneumology, teleradiology, teledermatology, telendoscopy, telepsychiatry, teleotolaryngology, teleophthalmology, and telecardiology [7]. The diversity of the telematic intervention used, ranged from simple telephone follow-up to sophisticated and comprehensive management teams composed of multiple members. "Telecare" is a form of telemedicine, and refers to medical/nursing management interventions made over the telephone. "Transtelephonic monitoring" refers to the transmission of physiologic variables over existing telephone lines to a network server to be displayed on a conventional personal computer. The transmission of physiologic variables (heart rate, blood pressure, weight, etc...) and biological signals (EKG trace, oximetry, spirometry etc...) provide objective data, which may indicate the need for medical/nursing intervention that are subsequently made over the telephone. The availability of objective physiologic data, trans-telephonically transmitted in real time, which can be coupled with the patient's subjective input, make the telemedicine method unique. The technology also adds to the cost-effectiveness and efficiency of the method.

\section{Application}

Various telemedicine approaches were reported in the literature. As telecommunication technology has advanced and costs have declined over the past decade, there has been a steady growth in telemedicine. Much of this growth, however, has been in the form of feasibility studies and pilot trials. The method used is completely different in every study. It is possible to distinguish two large categories of study methodologies: one in which telemedicine is an aid for helping the nurse in the management of patients with chronic diseases (Telenursing), and the other, completely automated, in which a computerised voice answering system and a computer algorythm checked the patient's vital signs with an acceptable range previously set by the physician (Telemanagement). The different approaches reported, presents different costs and different possibility of devices to be implemented and or different type of disease management to be applied. Indeed the different results obtained with apparently similar method are probably to ascribe also to the different quality of the technology applied.

However, the main problem in telemedicine is not a lack of technology; rather it is the lack of an organisational model able to capitalise the technology to improve healthcare. This is particularly important for the management of patients with chronic, disabling and relapsing disorders (congestive heat failure, chronic obstructive pulmonary disease, etc.) in which the possibility to send automatically different information to a call center and/or to receive a teleconsultation from a nurse or the own General Practitioner (GP) or a specialists could form together a new type of management with the lowest possible costs. A recent review about cost-effectiveness studies of telemedicine intervention [8], concludes that there is no good evidence that telemedicine is a cost-effective mean of delivering health care, even if all the actors appear quite completely satisfied by this new approach [9]. To reach more conclusive results, the need for better designed and conducted trials on chronic, disabling and demanding conditions, such as congestive heart failure, is emphasised.

We can distinguish different type of possible application of Telemedicine [10]: 


\section{Home telenursing}

Over the past decade there has been considerable interest in the possibility of using telemedicine as an aid in home nursing. Nurse-telephone triage lines have been running for over 10 years. Nurses answering the phones are specially trained and use algorithms on computers to steer the conversation to a particular conclusion [11]. Little is known about the effectiveness of nurses triaging by phone, probably because the schemes are not easy to assess. The data in the literature showed a benefit in terms of reduced hospital admissions and cost savings [12-16], but transposing the benefits to a wider population outside of a trial is not easy. Diagnosis and even prescribing by nurses remains an area of tension, especially as one barrier to the introduction of nurse phone-lines is doctors themselves, some of whom are suspicious and see their responsibilities being eroded. However, we believe that in a particular area, as in chronic disease, the nurses can manage patients in close collaboration with their GP and the specialists, as a part of an interdisciplinary disease.

Various feasibility studies into a range of different kinds of technology have been conducted with the aim to demonstrate that chronically ill patients can be provided with both cheaper and higher quality care than that provided by traditional home visit. Although these studies indicate that patient satisfaction is usually good [9], no clearcut evidence of cost-effectiveness has been obtained.

\section{Electronic referrals to specialists and hospitals}

The electronic referrals can be dealt with by the hospital staff, while the patient does not need to attend the outpatient clinic, either through electronic messages or by arranging a teleconsultation by video link. Another possible application for this model is the interaction between primary care or peripheral hospitals with greater and more specialised hospitals. In this case second opinions to doctors are provided by a panel of consultants that are not on site and may communicate with a teleconsultation. This approach is likely to be cost-effective [17-18].

\section{Teleconsulting between general practitioners and specialists}

Effective teleconsultation which takes place after the transmission of biological signals, between two professional figures, an experienced specialists and the GP; this aspect confirms the impression of the reciprocal reliance and compliance of GPs and specialists in managing problems and possibly in following diagnostic and therapeutic common protocols. Improving this professional relationship, Telemedicine can help to improve the appropriateness of hospital admissions and referrals to the Emergency Department, of the request of diagnostic tests and of chronic disease home management [19-25].

\section{Call centres and online health}

The growth in the number of telephone call centres that provide health information and advice shows that there is a request from the public for these services. Many of these call centres, such as National Health System (NHS) Direct [26], in England where the operators attempt to triage callers into those requiring emergency treatment, those who can be referred to primary care, and those who can be advised to treat themselves. Although there is reasonable evidence that these services are safe, little evidence exists that they reduce demand and costs for the NHS.

\section{Telepneumology}

As another field of medicine, pneumology can also be a really good field of application of Telemedicine. In the western world, epidemiological data shows that many respiratory disorders pose a huge load on each nation's health care system, for their increasing incidence and weight from social, psychological and economical points of view.

Due to their increasing incidence, Chronic obstructive pulmonary disease (COPD) and asthma, including the severe stages of both diseases when chronic respiratory failure develops, are clearly the two most important areas of interest for any health system willing to achieve a better cost-effect ratio. International guidelines call for a comprehensive management of chronic disabling disorders [27, 28]. In COPD, as an example, GOLD Guidelines emphasise an educational approach to the patients' need, able to reduce the costs (e.g. by reducing the rate of exacerbations) and at the same time to improve the quality of the services provided to the patients and their quality of life [27]. In addition, the COPD standards prescribe that once the COPD patient is discharged from the Hospital he/she should be followed, although there are no studies demonstrating which specific schedule of followup is more likely to achieve a better control of the disease management. An integrated care involving a team of primary and secondary care professionals is recommended, including an encouragement for the development of a system of patient selfmanagement and promotion of a healthy life-style.

Despite all of the above, a surprisingly low number of papers have been published on Telepneumology, at least partly due to the lack of evidence that this is a cost-effective approach. On the other hand, if no such studies are performed no clear-cut demonstration of the advantages (if any) of Telemedicine as an aid in the management of patients with respiratory diseases will ever be reached.

\section{ASTHMA}

In asthma, Pinnock's study [29] determined whether routine reviews by telephone of patients improve access and are good alternative to face to face reviews in general practice. Neither telephone reviews nor face to face reviews resulted in im- 
provement in asthma related quality of life or morbidity, and it may be tempting to conclude that routine reviews of asthma patients are ineffective. In spite of the results, the study could serve to describe a possible telephone intervention; telephone reviews were of shorter duration than the face to face consultations, though the content was similar, apart from practical procedures such as peak-flow measurements. The distribution of the timing of consultations suggest that the face to face consultations may have been paced to use the available 15,20 , or 30 minute appointments, whereas a telephone review could take as short or as long a time as needed. Time may be saved during a telephone review, as patients do not have to enter or leave the room, and computer templates and medical records can be completed during the course of the consultation. The nurses who undertook the reviews observed that the telephone consultations felt more "focused", which may reflect the recognised tendency for telephone interactions to be more goal oriented, with fewer digressions and achieving shared tasks faster. Patients' satisfaction was equally high with both modes of consultation. The nursing care satisfaction questionnaire included a domain that reflects "perceived time", and it is reassuring that despite the shorter duration of telephone consultations there was no evidence of dissatisfaction with the time spent.

Another study [30] was focused on children with asthma. A web-based store-and-forward teleconsultation asthma management system was implemented to provide asthma care to children of military families living in the Western Pacific Area. Children followed by teleconsultation had better outcomes such as improved inhaler technique, fewer ED visits and unscheduled visits for asthma. In a subsequent study, the same authors showed the effectiveness of an internet-based store-and-forward video home system to deliver asthma educational programmes, as compared to asthma education received by the patients through traditional clinic visits [31].

In the context of telemonitoring patients with asthma and respiratory diseases, Abboud described the possibility of monitoring patients' lung function at a remote centre, by using a portable spirometer that can transmit the data [32]. Also in this case the problem is not on the available ICT, but on their relevance and on the organisation of the Telemedicine service. The same authors in a subsequent paper described their experience with telespirometry control of 39 moderate to severe asthma patients and concluded that home monitoring may improve the quality of asthma management [33]. In the U.S.A., Finkelstein and co-workers studied the acceptance by the asthma patients from a low-income inner-city community of an internet based telemonitoring system. The patients were given portable spirometers and palmtop computers. They were able to exchange messages with a physician who reviewed their data. A pilot study was shown to provide reliable information [34] and a subsequent study showed that patients' compliance was higher in comparison with patients un- der standard care [35]. Outside urban areas, Romano and co-workers demonstrated that, even in a rural school-based health clinic, the implementation of a telemedicine system improved asthma symptoms and quality of life [36]. Rural Arkansas was the scenario for another interesting study in asthma patients. Bynum and Colleagues described their system of telepharmacy counselling, providing accessible education regarding metered-dose inhalers (MDI) technique for adolescents. Results indicated that the telepharmacy followed patients had an improvement in their MDI technique [37]. Outside the U.S.A., similar experiences with asthma patients followed by telemedicine were described in UK [38], in Romania [39] and in Japan [40].

\section{COPD}

Although a telecommunications system has not yet been employed in the management of patients with COPD, a few published trials [41-44] have addressed the potential impact of interventions that improve the monitoring of patients with COPD and their adherence to their treatment regimen. Despite certain methodological limitations, including small sample size, these studies have shown that COPD monitoring interventions have appeared to reduce either health-care utilisation or mortality rates in COPD patients. A reduction in $50 \%$ costs for emergency services and total expenses for general practice was reached in a randomised controlled trial evaluated an inpatient education programme for patient admitted to the hospital for COPD [41]. Similar results were reported by Littlejohn [42] in a randomised controlled trial concerning the effectiveness of an outpatient respiratory-care worker who provided patient education, symptom monitoring and treatment monitoring and who served as a liaison between primary care providers and hospital services. In the intervention group there was a reduction in mortality rate, in the sickness impact profile scores and in the indexes of impairment. Cockcroft [43] conducted a randomised controlled trial in patients with advanced COPD, evaluating the effectiveness of monthly home visit by a nurse who provided patients with education in adherence with therapeutic regimens and the early self detection of clinical deterioration. A project with the application of a telephone-linked computer system for COPD care is described by Young [44], but the results are not yet published.

Cost and effectiveness data from the Milwaukee and Iron Mountain Veterans Affairs Medical Centers (VAMC) telepulmonary programme [45] were collected for a period of 1 year. The cost-effectiveness analysis was conducted from a societal perspective. Average and incremental cost-effectiveness ratios were calculated together with sensitivity analysis. Telemedicine was found to be more cost effective (\$335 per patient/year) compared to routine care (\$585 per patient/year) and on-site care (\$1166 per patient/year). Sensitivity analysis revealed that cost effectiveness of telemedicine 
was sensitive to changes in the values for the number of patients, probability of successful telemedicine consultation, telemedicine equipment cost, utility of telemedicine, and percentage effort assigned to the on-site pulmonary physician. Telemedicine is a cost-effective alternative for the delivery of outpatient pulmonary care for rural populations with limited access to subspecialty services. Cost effectiveness of telemedicine is related to three major factors: cost sharing, i.e., adequate patient volume and sharing of telemedicine infrastructure amongst various clinical users; effectiveness of telemedicine in terms of patient utility and successful clinical consultations; and indirect cost savings accrued by decreasing cost of patients' lost productivity.

Kaiser Permanente Organisation [46] recently reported the first formal randomised controlled trial of home videophones. In this trial patients newly diagnosed with a chronic condition were nursed at home. Patients in the intervention group were equipped with home videophones, an electronic stethoscope and a digital blood pressure monitor; over 18 months, patients in the telemedicine group received $17 \%$ fewer home visits by nurses than control group and the average cost of care in this group was $27 \%$ less than in the control group. The patients receiving telemedicine were pleased with the equipment and were nursed as effectively as the control group.

Other data published on Telemedicine in patients with COPD describe a randomised controlled trial of home telecare in COPD exacerbations [47], suggesting an improvement of their management through the use of telemedicine, and the implementation of an internet-based telematic service for patients with severe and chronic diseases, including COPD [48].

\section{TELEMEDICINE APPLICATIONS TO OTHER RESPIRATORY DISEASES}

The application of telemedicine to Obstructive Sleep Apnea (OSAS) patients has been studied. Starting from the concept that OSAS is a typical "life-style" disease, Oki and Colleagues evaluated telemedicine as an aid in OSAS patients to deliver home-based education on nutrition and exercise [49]. Evaluating the possibility that remote monitoring could be an alternative to sleep laboratory studies Escourrou and co-workers performed a validation study, and concluded that there is a need for such an approach to decrease costs and to match the increasing request of polysomnography [50]. The comparison between a home unattended and a telemonitored polysomnography was performed by Gagnadoux and Colleagues. They studied in a crossover trial 99 patients who underwent polysomnography with both modalities and concluded that telemonitored polysomnography is clearly superior to home unattended polysomnography from a technical point of view and tends to be preferred by patients. Noticeably, the site of recording (home vs hospital) has no influence on polysomnographic indexes [51]. Another impor- tant possible application of telemedicine for patients with OSAS is the continuous positive airway pressure (CPAP) monitoring. Lankford examined the accuracy and reproducibility of transmitting CPAP compliance and usage data using a novel wireless and internet technology. The study included 30 patients who were asked to transmit the data and to download the data from the flow generator for comparative purposes. The data confirmed a total agreement between transmitted and downloaded data, demonstrating the reliability of the wireless system and suggesting potential benefits of this technology in this field [52].

Other interesting applications of telemedicine include the possibility of increasing the potential of a respiratory clinic to serve more patients by implementing a telephone and e-mail consultation [53], home telemonitoring for patients with chronic respiratory failure [54], the possibility of improving efficiency of ICU using telemedicine [55] and the transmission though internet of histopathologic images as a second opinion from pathologists who are experts in the field of respiratory diseases [56].

Finally, is should be noted that in a Position Statement the American Association of Cardiovascular and Pulmonary Rehabilitation aimed at describing the standards, ethics, credentials, liability and reimbursement considerations on Telemedicine, it is declared that Telemedicine can be used as 1) an adjunctive therapeutic modality; 2) a method to extend the time frame of therapeutic contact; 3) an alternative when in-person contact is not possible [57].

\section{Conclusions}

This preliminary data confirms the efficacy and soundness of a telemedicine project [58]. For this reason these projects are entered in the general view of the multidisciplinary programs for the management of chronic disease. While the intervention trial have utilised different designs, the nurse practitioner is common to all the trials and appears to have a key role in the management of chronic disease. The different approaches (multidisciplinary home-based, integrated, telematic) present different costs and possibilities to be implemented. For example, structured home visits can be applied to patients living around the hospital. On the contrary a telematic approach allows the patient to contact the service at anytime and anywhere. Furthermore the number of patients involved is potentially enormous. Besides the advantages of an early diagnosis and a tailored therapeutic intervention in the home management of chronic illnesses, further potentials of telecare are the availability of specialist teleconsultation out of hospital, an improvement in appropriateness of hospital admissions and referrals to the Emergency Department. An integrated management involving the patient, his family, the primary and secondary care with a telematic approach or home visits following the different patient's clinic situation will be desirable. Further studies are obviously re- 
quired to develop these management strategies further. It should be noted that all the management programmes have only been applied to patients who have been admitted to hospital. Consequently, there is no evidence of the benefits in reducing the first admission for patients with newly diagnosed pulmonary disease. In addition the programmes have generally only followed patients for a relatively short period and the long term effect remain uncertain. In particular, if the cost/effective analysis also proves to be advantageous, we could foresee, the spread of an internet network, linking patients, General Practitioners and hospitals and ambulatory specialists for the management of chronic disease with probably the result to improve the quality of life of the patients and to reduce the public health burden of this disease.

\section{References}

1. Saxena SC, KumarV, Giri VK. Telecardiology for effective health care services. Journal of medical engineering \& technology 2003; 27: 149-158.

2. Ganapathy k., 2000, telemedicine in India - the Apollo experience. Http: //www. Thamburaj. Com/telemedicine.htm.

3. Fisk NM. Foetal telemedicine: interactive transfer of real time ultrasound and video via isdn for remote consultation. J Telemed Telecare 1995; 1, 38-45.

4. Ingenerf J. Telemedicine and terminology: different needs of context information. IEEE transactions on information technology in biomedicine 1999; 3, 92-100.

5. General accounting office. Telemedicine: federal strategy is needed to guide investments. GAO Washington 1997.

6. Lin JC. Applying telecommunication technology to health-care delivery. IEEE engineering in medicine and biology 1999, 18, 28-31.

7. Priadarsini JB. Teleconsultation for healthcare. M. Tech. Dissertation, electrical engineering department, IIT Roorkee. 2002.

8. Whitten SP, Mair SF, Haycox A, May RC,Williams TL, Hellmich S. Systematic review of cost effectiveness studies on telemedicine interventions. $\mathrm{Br}$ Med $\mathrm{J}$ 2002; 324: 1434.

9. Mair F, Whitten P. Systematic review of studies of patients satisfaction with telemedicine. Br Med J 2000; 320: $1517-1520$.

10. Wootton R. Telemedicine: Clinical review. Br Med J 2001; 323: 557-60.

11. Nurse telephone-triage. The Lancet 2001; 357 (9253): 323.

12. Heidenreich PA, Ruggerio CM, Massie BM. Effect of a home monitoring system on hospitalization and resource use for patients with heart failure. Am Heart $J$ 1999; 138: 633-640.

13. Bondmass M, Bolger N, Castro G, Avitall B. A prospective randomized study comparing outcomes and outpatient care delivery method for chronic heart failure. JACC 2001; 37 Suppl A: 1A-648A.

14. Shah NB, Der E, Ruggerio C, Heidenreich PA, Massie BM. Prevention of hospitalisations for heart failure with an interactive home monitoring program. Am Heart J 1998; 135: 373-378.

15. De Lusignan $\mathrm{S}$, Wells $\mathrm{S}$, Johnson $\mathrm{P}$, Meredith $\mathrm{K}$, Leatham E. compliance and effectiveness of 1 year's home telemonitoring. The report of a pilot study of patient with chronic heart failure. European J Heart Failure 2001; 3: 723-730.

16. Riegel B, Carlson B, Kopp Z, Le Petri B, Glaser D,
Unger A. Effect of a standardised nurse case-management telephone intervention on resource use in patient with chronic heart failure. Arch Inter Med 2002; 162: 705-12.

17. Harno K, Paavola T, Carlson C et al. Patient referral by telemedicine: effectiveness and cost analysis of an intranet system. J Telemed Telecare 2000; 6: 320-329.

18. Vassallo DJ, Hoque F, Farquarson Roberts M, Patterson V, Swinfen P, Swinfen R. An evaluation of the first year's experience with a low cost telemedicine link in Bangladesh. J Telemed Telecare 2001; 7: 125-138.

19. Scalvini S, Zanelli E, Domenighini D, et al. Telecardiology community: a new approach to take care of cardiac patients. Cardiologia 1999; 44: 921-24.

20. Scalvini S, Zanelli E, Gritti M, Pollina R, Giordano A, Glisenti F. Appropriatezza diagnostica dell'invio in pronto soccorso mediante un servizio di telecardiologia. Ital Heart J 2000; 1: 905-909.

21. Scalvini S, Zanelli E, Volterrani M, Castorina M, Giordano A, Glisenti F. Riduzione potenziale dei costi per il Servizio Sanitario Nazionale mediante un servizio di telecardiologia dedicato ai medici di medicina generale. Ital Heart J 2001: 2 (Suppl): 1091-1097.

22. Scalvini S, Zanelli E, Conti C, et al. Assessment of prehospital chest pain using telecardiology. J Telemed Telecare 2002; 8 (4): 231-6.

23. Hallam L, Cragg D. Organization of primary care services outside normal working hours. Br Med J 1994; 309: 1621-1623.

24. Olsen F, Jolleys JV. Out of hours services: the Danish solution examined. Br Med J 1994; 309: 1624-26.

25. Wallace P, Haines A, Harrison R, et al. Joint teleconsultation (virtual outreach) versus standard outpatient appointments for patients referred by their general practitioner for a specialist opinion: a randomised trial. Lancet 2002; 359: 1961-68.

26. Munro J, Nicholl J, O'Cathain A, Knowles E. Impact of NHS Direct on demand for immediate care: observational study. Br Med J 2000; 321: 150-153.

27. Pauwels RA, Buist AS, Calverley PM, Jenkins CR, Hurd SS. The GOLD Scientific Committee. Global strategy for the diagnosis, management, and prevention of chronic obstructive pulmonary disease. NHLBI/ WHO Global Initiative for Chronic Obstructive Lung Disease (GOLD) Workshop Summary. Am J Resp Crit Care Med 2001; 163:1256-1276.

28. www.ginasthma.com. Accessed jan $31^{\text {st }} 2005$.

29. Pinnock H, Bawden R, Proctor R, et al. Accessibility, acceptability and effectiveness in primary care of routine telephone review of asthma: pragmatic, randomised controlled trial. Br Med J 2003; 326: 477-9.

30. Malone F, Callahan CW, Chan DS, Sheets MS, Person DA. Caring for children with asthma through teleconsultation: "ECHO-Pac", the electronic children's hospital of the Pacific. Telemed J E Health 2004; 10: 138-146.

31. Chan DS, Callahan CW, Sheets SJ Moreno CN, Malone FJ. An internet-based store-and-forward video home telehealth system for improving asthma outcomes in children. Am J Health-Syst Pharm 2003; 60: 1976-81.

32. Abboud S, Bruderman I. Assessment of a new transtelephonic portable spirometer. Thorax 1996; 51: 407-10.

33. Bruderman I, Abboud S. Telespirometry: novel system for home monitoring of asthma patients. Telemed $J$ 1997; 3: 127-33.

34. Finkelstein J, Hripcsak G, Cabrera MR. Patients' acceptance of Internet-based asthma telemonitoring. Proc. AMIA Symp 1998; 336-40.

35. Finkelstein J, O'Connor G, Friedmann RH. Development and implementation of the home asthma telemonitoring (HAT) system to facilitate asthma self-care. Medinfo 2001; 10 (Pt 1): 810-4. 
36. Romano MJ, Hernandez J, Gaylor A, Howard S, Knox R. Improvement in asthma symptoms and quality of life in pediatric patients through speciality care delivered via telemedicine. Telemed J E Health 2001; 7: 281-6.

37. Bynum A, Hopkins D, Thomas A, Copeland N, Irwin C. The effect of telepharmacy counseling on metereddose inhaler technique among adolescents with asthma in rural Arkansas. Telemed J E Health 2001; 3: 207-17.

38. Steel S, Lock S, Johnson N, Martinez Y, Marquilles E, Bayford R. A feasibility study of remote monitoring of asthmatic patients. J Telemed Telecare 2002; 8: 290-6.

39. Dobre I, Croitoriu MG, Basca N, Oraseanu D. ASITASTM telematic assistance for chronic asthma. Stud Health Technol Inform 2002; 90: 201-5.

40. Kokubu F, Suzuki H, Sano Y, Kihara N, Adachi M. Telemedicine system for high-risk asthmatic patients. Aerugi 1999; 48: 700-12.

41. Haggerty Mc, Stockdale- Woolley R, Nair S. Respicare: an innovative home care program for the patient with chronic obstructive pulmonary disease. Chest 1991; 100: 607-612

42. Littlejohns P, Baveystock $\mathrm{Cm}$, Parnell $\mathrm{H}$, et al. Randomized controlled trial of the effectiveness of a respiratory health worker in reducing impairment, disability, and handicap due to chronic airflow limitation. Thorax 1991; 46: 559-564

43. Cockcroft A, Bagnall P, Heslop A, et al. Controlled trial of respiratory health worker visiting patients with chronic respiratory disability. Br Med J 1987; 294: 225228

44. Young M, Sparrow D, Gottlieb D, Mph; Selim A, Friedman R. A telephone- linked computer system for COPD care. Chest 2001; 119: 1565-1575.

45. Zia Agha, Ralph M. Schapira, Azmaira H. Maker. Cost effectiveness of telemedicine for the delivery of outpatient Pulmonary care to a rural population. Telemed $J E$ Health 2002; 8: 281-291.

46. Johnston B, Wheeler L, Deuser J, Sousa KH. Outcomes of the Kaiser Permanente Tele-Home Health Research Project. Arch Fam Med 2000; 9: 40-45.
47. Mair FS, Boland A, Angus R, et al. A randomized controlled trial of home telecare. J Telemed Telecare 2002; 8 (suppl 2): 58-60.

48. Stalidis G, Prentza A, Vlachos IN, Maglavera S, Koustouris D. Medical support system for continuation of care based on XML web technology. Int J Med Inform 2001; 64: 385-400.

49. Oki Y, Shiomi T, Sasanabe R et al. Multiple cardiovascular risk factors in obstructive sleep apnea syndrome patients and an attempt at lifestyle modification using telemedicinebased education. Psichiatry Clin Neurosci 1999; 53: 311-3.

50. Escourrou P, Luriau S, Rehel M, Nedelcoux H, Lanoe JL. Needs and costs of sleep monitoring. Stud Health Technol Inform 2000; 78: 69-85.

51. Gagnadoux F, Pelletier-Fleury N, Philippe C, Rakotonanahary D, Fleury B. Home unattended vs. hospital telemonitored polysomnography in suspected obstructive sleep apnea syndrome: a randomized crossover trial. Chest 2002;121: 753-8.

52. Lankford DA. Wireless CPAP patient monitoring: accuracy status. Telemed J E Health 2004; 10: 162-9.

53. Partridge MR. An assessment of the feasibility of telephone and email consultation in a chest clinic. Patient Educ Couns 2004 Jul; 54: 11-3.

54. Maiolo C, Mohamed EI, Fiorani CM, De Lorenzo A Home tele-monitoring for patients with severe respiratory illnesses: the Italian experience. $J$ Telemed Telecare 2003; 9: 67-71.

55. Chapman M,Gattas D, Suntharalingam G. Innovations in technology for critical care medicine. Crit Care 2004; 8: 74-6.

56. Leong FJ, Nicholson AG, McGee JO. Robotic telepathology: efficacy and usability in pulmonary pathology. J Pathol 2002; 197: 211-7.

57. Shaw K, Heggestad-Hereford JR, Southard DR, Sparks KE. American Association of Cardiovascular and Pulmonary Rehabilitation Telemedicine Position Statement. J Cardiopulm Rehab 2001, 21: 261-2.

58. Hailay D, Roine R,Ohinmaa A. Systematic review of evidence for the benefits of telemedicine. $J$ Telemed Telecare 2002; 8 (suppl 1): 1-7.

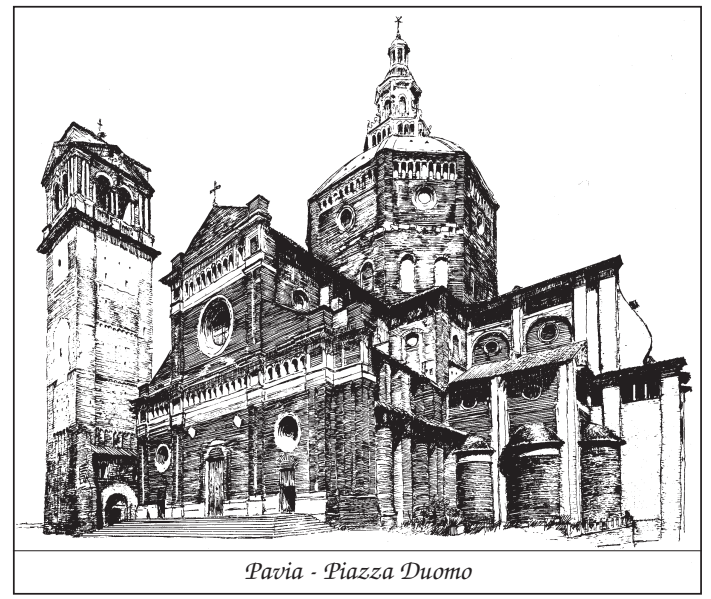

